\title{
Symmetric Harmonic Maps Between Spheres
}

\author{
Wei-Yei Ding
}

Institute of Mathematics, Academia Sinica, Beijing, People's Republic of China

\begin{abstract}
Necessary and sufficient conditions for the existence of symmetric harmonic maps between spheres are established.
\end{abstract}

\section{Introduction}

In [S], Smith studied the problem of existence of harmonic maps between Euclidean spheres. The harmonic maps he constructed are of very special type. Starting from two homogeneous harmonic polynomial maps $f: S^{p} \rightarrow S^{m}$ of homogeneity $k$ and $g: S^{q} \rightarrow S^{n}$ of homogeneity $l$, Smith sought for harmonic maps from $S^{p+q+1}$ into $S^{m+n+1}$ which are of the following form:

$$
u(x, y)=\left(\sin a(t) f\left(\frac{x}{|x|}\right), \cos a(t) g\left(\frac{y}{|y|}\right)\right),
$$

where $x \in R^{p+1}, y \in R^{q+1}$, with $|x|^{2}+|y|^{2}=1, t=\log (|x| /|y|)$, and $a(t)$ is a real function with range in $\left[0, \frac{\pi}{2}\right]$. It is proved that if $a(t)$ satisfies the following equation:

$$
\ddot{a}+\frac{(p-1) e^{-t}-(q-1) e^{t}}{e^{t}+e^{-t}} \dot{a}+\frac{\lambda_{2} e^{t}-\lambda_{1} e^{-t}}{e^{t}+e^{-t}} \sin a \cos a=0
$$

together with the conditions

$$
0 \leqq a(t) \leqq \frac{\pi}{2}, \quad \forall t \in R,
$$

and

$$
\lim _{t \rightarrow-\infty} a(t)=0, \quad \lim _{t \rightarrow \infty} a(t)=\frac{\pi}{2}
$$

then the map defined in (1.1) is actually an analytic harmonic map. Here $\lambda_{1}=k(k+p-1)$ and $\lambda_{2}=l(l+q-1)$. It is obvious that such a map is homotopic to the join $f * g$ of $f$ and $g$ [which is defined by (1.1) with $\sin a(t)=|x|$ and $\cos a(t)=|y|]$.

Smith proved that if $(p-1)^{2}<4 \lambda_{1}$ and $(q-1)^{2}<4 \lambda_{2}$ or $p=q$ and $\lambda_{1}=\lambda_{2}$, then there exists a harmonic map homotopic to $f * g$. Quite recently, Ratto [R] showed that the same conclusion holds provided $\lambda_{1}=p \leqq 5$. In this paper, we completely solve the problem of existence of harmonic maps of Smith's type. Our main result is the following: 
Theorem. Assume that

$$
\lambda_{1}(q-1) \geqq \lambda_{2}(p-1) .
$$

For the existence of a harmonic map of the form (1.1) it is necessary and sufficient that either (i) $(q-1)^{2}<4 \lambda_{2}$, or (ii) $(q-1)^{2} \geqq 4 \lambda_{2}$, and

$$
\sqrt{(p-1)^{2}+4 \lambda_{1}}+\sqrt{(q-1)^{2}-4 \lambda_{2}}<p+q-2 \text {. }
$$

One should note that (1.2) is not a restriction, since it can always be assumed by interchanging the roles of $f$ and $g$ whenever necessary.

We will use variational methods to prove Theorem 1.1. For the existence part of the theorem, essentially we obtain our harmonic maps by minimizing the energy over the class of mappings of the form (1.1). The only difficulty here is to show the energy-minimizing map is different from two specific weakly harmonic maps [corresponding to $a(t) \equiv 0$ and $\frac{\pi}{2}$ in (1.1)]. This is overcome by a complete analysis of the stability of the two weakly harmonic maps as critical points of the energy. The proof of the nonexistence part of the theorem seems more interesting, since it relies on the mountains pass theorem which is usually used for existence proof.

We prove Theorem 1.1 in the next section. In Sect. 3, we briefly discuss some easy consequences of Theorem 1.1, i.e. some new examples of symmetric harmonic maps between spheres.

\section{Proof of Theorem 1.1}

We first change the equation in Sect. 1 into a more convenient form. By a change of variable: $t \mapsto \log \operatorname{tg} t$, the equation is transformed into

$$
\ddot{a}+(p \operatorname{ctg} t-q \operatorname{tg} t) \dot{a}+\left(-\frac{\lambda_{2}}{\cos ^{2} t}-\frac{\lambda_{1}}{\sin ^{2} t}\right) \sin a \cos a=0,
$$

while the corresponding conditions become

$$
\begin{gathered}
0 \leqq a(t) \leqq \frac{\pi}{2}, \quad t \in\left(0, \frac{\pi}{2}\right), \\
\lim _{t \rightarrow 0} a(t)=0, \quad \lim _{t \rightarrow \frac{\pi}{2}} a(t)=\frac{\pi}{2},
\end{gathered}
$$

Throughout this section we assume that $p, q \geqq 1$ and $\lambda_{1}, \lambda_{2}>0$ are real parameters. Set $f(t)=\sin ^{p} t \cos ^{q} t$. It is easy to see that (2.1) is the Euler-Lagrange equation of the functional:

$$
J(a)=\int_{0}^{\frac{\pi}{2}}\left(\dot{a}^{2}+\frac{\lambda_{1} \sin ^{2} a}{\sin ^{2} t}+\frac{\lambda_{2} \cos ^{2} a}{\cos ^{2} t}\right) f(t) d t .
$$

It is not surprising that, up to a constant factor, $J(a)$ is just the energy of the mapping $u$ defined by (1.1).

$$
\text { Let } X=\left\{a \in H_{\mathrm{loc}}^{1}\left(0, \frac{\pi}{2}\right):\|a\|^{2}=\int_{0}^{\frac{\pi}{2}}\left(\dot{a}^{2}+a^{2}\right) f d t<\infty\right\} \text {. It is clear that with the inner }
$$

product

$$
(a, b)=\int_{0}^{\frac{\pi}{2}}(\dot{a} \dot{b}+a b) f d t
$$


$X$ is a Hilbert space. We remark that if $p>1$ and $q>1$, then $J$ is well defined and smooth on $X$. This is due to the following weighted Sobolev inequality:

$$
\int_{0}^{\frac{\pi}{2}} \frac{a^{2}}{s^{2}(t)} f(t) d t \leqq C\|a\|^{2}, \quad \forall a \in X,
$$

where $s(t)=\sin t$ (or $\cos t$ ). However, if $p=1$ (or $q=1$ ) the inequality fails, and $J$ is even not everywhere defined on $X$. In such a case, we still view $J$ as a functional on $X$ by allowing it to take values of $+\infty$. In view of condition (2.2), we want to minimize $J$ over the closed convex subset

$$
X_{0}=\left\{a \in X: 0 \leqq a(t) \leqq \frac{\pi}{2}, \forall t \in\left(0, \frac{\pi}{2}\right)\right\} .
$$

Lemma 2.1. There exists $a_{0} \in X_{0}$ which minimizes $J$ over $X_{0}$, i.e.

$$
J\left(a_{0}\right)=c_{0} \equiv \inf \left\{J(a): a \in X_{0}\right\} .
$$

Furthermore, if $a_{0} \neq 0$ or $\frac{\pi}{2}$, then $a_{0}$ satisfies (2.1)-(2.3).

Proof. It is not hard to show the existence of $a_{0}$ by minimizing $J$ directly over $X_{0}$. However, then we have to prove $a_{0}$ is actually a critical point of $J$ on $X$, i.e. it is a solution of (2.1). This causes some trouble. It will be more convenient to use the following trick.

For any $a \in X$, define $a^{*} \in X_{0}$ by

$$
a^{*}(t)=\left\{\begin{array}{lll}
\frac{\pi}{2}, & \text { if } & a(t)>\frac{\pi}{2} \\
a(t), & \text { if } & 0 \leqq a(t) \leqq \frac{\pi}{2} \\
0, & \text { if } & a(t)<0
\end{array}\right.
$$

Next, define $G: R \times R \rightarrow R$ by

$$
G(t, a)= \begin{cases}F\left(t, \frac{\pi}{2}\right), & \text { if } \quad a>\frac{\pi}{2} \\ F(t, a), & \text { if } \quad 0 \leqq a \leqq \frac{\pi}{2} \\ F(t, 0), & \text { if } \quad a<0\end{cases}
$$

where

$$
F(t, a)=\frac{\lambda_{1} \sin ^{2} a}{\sin ^{2} t}+\frac{\lambda_{2} \cos ^{2} a}{\cos ^{2} t}
$$

Let $J^{*}(a)=\int_{0}^{\frac{\pi}{2}}\left(\dot{a}^{2}+G(t, a)\right) f(t) d t$. Note that $F\left(t, a^{*}(t)\right)=G(t, a(t))=G\left(t, a^{*}(t)\right)$ for all $a \in X$ and $t \in\left(0, \frac{\pi}{2}\right)$. It follows that

$$
J\left(a^{*}\right)=J^{*}\left(a^{*}\right) \leqq J^{*}(a), \quad \forall a \in X .
$$

This implies $\inf \left\{J^{*}(a): a \in X\right\}=c_{0}$. Now let $\left\{a_{i}\right\}$ be a minimizing sequence in $X$ for $J^{*}$. By passing to $a_{i}^{*}$ if necessary, we may assume $a_{i} \in X_{0}$. Thus, since $J *\left(a_{i}\right) \rightarrow c_{0}$ and $a_{i}$ satisfies (2.2), we see that $\left\{a_{i}\right\}$ is bounded in $X$. It follows that some subsequence of $\left\{a_{i}\right\}$ converges weakly in $X$ to some $a_{0} \in X_{0}$. It is routine to verify that the functional $J$ is weak-lower-semi-continuous on $X$, and consequently $J^{*}\left(a_{0}\right)=c_{0}$, i.e. $a_{0}$ achieves the infimum of $J^{*}$ on $X$. Therefore, $a_{0}$ satisfies the Euler-Lagrange 
equation for $J^{*}$. Since $a_{0} \in X_{0}$, i.e. $a_{0}$ satisfies (2.2), the equation satisfied by $a_{0}$ is exactly (2.1) due to the fact that $J^{*}$ and $J$ coincide on $X_{0}$. Now, if $a_{0} \neq 0$ or $\frac{\pi}{2}$, then by Lemma 5.5 of [S], $a_{0}$ also satisfies (2.3). (Note the condition that $|\dot{a}| \leqq C$ in Lemma 5.5 of $[\mathrm{S}]$ is unnecessary, since it is implied by Lemma 5.1 in [H].) This completes the proof of Lemma 2.1.

As an immediate consequence of Lemma 2.1 we have

Lemma 2.2. If $c_{0}<\min \left\{J(0), J\left(\frac{\pi}{2}\right)\right\}$, then there exists a solution to (2.1)-(2.3).

By a direct computation, we see the assumption (1.2), which will be assumed throughout this section, is equivalent to $J(0) \leqq J\left(\frac{\pi}{2}\right)$. Thus, the condition $c_{0}<\min \left\{J(0), J\left(\frac{\pi}{2}\right)\right\}$ in Lemma 2.2 can be simplified to $c_{0}<J(0)$. We will verify this condition through considerations of the second variation of $J$ at the constant critical point 0 :

$$
2 I(v)=d^{2} J(0)(v, v)=2 \int_{0}^{\frac{\pi}{2}}\left(\dot{v}^{2}+Q(t) v^{2}\right) f(t) d t,
$$

where $Q(t)=\lambda_{1} \csc ^{2} t-\lambda_{2} \sec ^{2} t$.

Lemma 2.3. If there exists $v \in X$ such that $v \geqq 0$ and $I(v)<0$, then $c_{0}<J(0)$.

Proof. We may assume $q>1$, since otherwise $q=1$ and $J(0)=\infty$, the lemma is trivially true. Let $v_{M} \in X$ be such that $v_{M}(t)=v(t)$ if $v(t)<M$, and $v_{M}(t)=M$ if $v(t) \geqq M$. Then one checks easily that $I\left(v_{M}\right) \rightarrow I(v)$ as $M \rightarrow \infty$. It follows that $I\left(v_{M}\right)<0$ for sufficiently large $M$. Fixing such a large $M$, we have

$$
\left.\frac{d}{d s} J\left(s v_{M}\right)\right|_{s=0}=0 \text { and }\left.\frac{d^{2}}{d s^{2}} J\left(s v_{M}\right)\right|_{s=0}=2 I\left(v_{M}\right)<0 \text {. }
$$

Therefore, $J\left(s v_{M}\right)<J(0)$ for small $s>0$. But $v_{M}$ is nonnegative and bounded, so $s v_{M} \in X_{0}$ for small $s>0$. It follows from the definition of $c_{0}$ that $c_{0}<J(0)$.

Lemma 2.4. If $(q-1)^{2}<4 \lambda_{2}$, then $c_{0}<J(0)$.

Proof. Let $v=\sin ^{s} t \cos ^{-r} t$, where $s>0$ and $r<(q-1) / 2$. Then $v \in X$ and $v \geqq 0$. A direct computation shows

$$
I(v)=C_{1}\left[\left(q^{2}-4 r-1-4 \lambda_{2}\right) s^{2}+C_{2} s+C_{3}\right],
$$

where $C_{1}>0$, and $C_{2}, C_{3}$ do not depend on $s$. If $(q-1)^{2}<4 \lambda_{2}$, we may choose $r$ close to $(q-1) / 2$ so that the coefficient of $s^{2}$ is negative. Then letting $s$ be sufficiently large, we see $I(v)<0$. It follows from Lemma 2.3 that $c_{0}<J(0)$.

Next, we consider the case where $(q-1)^{2} \geqq 4 \lambda_{2}$. For this case we have to solve an eigenvalue problem as follows. Let

$$
\mu_{0}=\inf \left\{I(v): v \in X, \int_{0}^{\frac{\pi}{2}} v^{2} f(t) d t=1\right\} .
$$

Formally, if $\mu_{0}$ is finite and some $v_{0} \in X$ achieves the value $\mu_{0}$, then $v_{0}$ is a solution of the following Euler-Lagrange equation:

$$
-\frac{d}{d t}(f(t) \dot{v})+Q(t) v=\mu f(t) v
$$


where the constant $\mu$ appears as a Lagrange multiplier. Our method to determine the exact value of $\mu_{0}$ relies on the fortunate fact that we can explicitly solve (2.5) with some $v_{0} \in X$ and constant $\mu$. Then we are able to show $\mu=\mu_{0}$.

Set $v_{0}=\sin ^{s} t \cos ^{-r} t$, where

$$
s=\frac{1}{2}\left[\sqrt{(p-1)^{2}+4 \lambda_{1}}-(p-1)\right], \quad r=\frac{1}{2}\left[(q-1)-\sqrt{(q-1)^{2}-4 \lambda_{2}}\right] .
$$

Note that $s>0$ and $r<(q-1) / 2$, so $v_{0} \in X$. One can easily verify that $v_{0}$ satisfies Eq. (2.5) with the constant

$$
\mu=(r-s)^{2}-(r-s)(p+q) .
$$

Lemma 2.5. If $(q-1)^{2} \geqq 4 \lambda_{2}$ and

$$
\sqrt{(p-1)^{2}+4 \lambda_{1}}+\sqrt{(q-1)^{2}-4 \lambda_{2}}<p+q-2,
$$

then $c_{0}<J(0)$.

Proof. It is easy to verify $I\left(v_{0}\right)=\mu \int_{0}^{\frac{\pi}{2}} v_{0}^{2} f(t) d t$. Thus, $I\left(v_{0}\right)<0$ iff $\mu<0$. But it is also easy to see that $\mu<0$ is equivalent to (2.6). Hence, if (2.6) holds, then we have $I\left(v_{0}\right)<0$ and the lemma follows from Lemma 2.3.

Combining Lemmas 2.2, 2.4, and 2.5, we conclude that if the condition of Theorem 1.1 is satisfied, then there exists a solution to (2.1)-(2.3). By Smith ([S]), this solution gives rise to an analytic harmonic map of the form (1.1). This completes the proof of sufficiency part of Theorem 1.1.

It remains to prove the necessary part of Theorem 1.1. We have to show if $(q-1)^{2} \geqq 4 \lambda_{2}$ while (2.6) does not hold, then no solutions can exist. In such a case, we know from the proof of Lemma 2.5 that $\mu \geqq 0$.

Lemma 2.6. $\mu_{0}=\mu$.

$\frac{\pi}{2}$

Proof. Note first that since $I\left(v_{0}\right)=\mu \int_{0}^{\frac{\pi}{2}} v_{0}^{2} f d t$, we have $\mu \geqq \mu_{0}$ by the definition of $\mu_{0}$. We need only to show the converse.

For small $\varepsilon>0$, let $H_{\varepsilon}$ be the subspace of $X$ consisting of functions with supports in $\left[\varepsilon, \frac{\pi}{2}-\varepsilon\right]$. Let

$$
\mu_{\varepsilon}=\inf \left\{I(v): v \in H_{v}, \int_{0}^{\frac{\pi}{2}} v^{2} f(t) d t=1\right\} .
$$

Since on $\left[\varepsilon, \frac{\pi}{2}-\varepsilon\right] Q(t)$ is bounded, one proves in a standard way that $\mu_{\varepsilon}$ is achieved by some $v_{\varepsilon} \in H_{\varepsilon}$ which satisfies

$$
\begin{aligned}
-\frac{d}{d t}\left(f(t) \dot{v}_{\varepsilon}\right)+Q(t) f(t) v_{\varepsilon} & =\mu_{\varepsilon} f(t) v_{\varepsilon}, \quad v_{\varepsilon}>0, \text { on }\left(\varepsilon, \frac{\pi}{2}-\varepsilon\right), \\
v_{\varepsilon}(\varepsilon) & =v_{\varepsilon}\left(\frac{\pi}{2}-\varepsilon\right)=0 .
\end{aligned}
$$

We claim $\mu_{\varepsilon}>\mu$. Indeed, multiplying Eqs. (2.5) (with $v=v_{0}$ ) and (2.7) by $v_{\varepsilon}$ and $v_{0}$ respectively and integrating by parts leads to

$$
\left(\mu_{\varepsilon}-\mu\right) \int_{\varepsilon}^{\frac{\pi}{2}} v_{0} v_{\varepsilon} f(t) d t=\left.f \dot{v}_{0} \dot{v}_{\varepsilon}\right|_{\frac{\pi}{2}-\varepsilon} ^{\varepsilon}>0 .
$$


This is because $v_{\varepsilon}$ is positive in the interior of the interval and it is zero at the endpoints, hence $\dot{v}_{\varepsilon}(\varepsilon)>0$ and $\dot{v}_{\varepsilon}\left(\frac{\pi}{2}-\varepsilon\right)<0$. Since the integral on the left is positive, we see $\mu_{\varepsilon}>\mu$.

Next, we claim that

$$
\mu_{\varepsilon} \rightarrow \mu_{0}, \quad \text { as } \quad \varepsilon \rightarrow 0 .
$$

If (2.8) holds, then letting $\varepsilon \rightarrow 0$ in the inequality $\mu_{\varepsilon}>\mu$, we get $\mu_{0} \geqq \mu$, completing the proof. To show (2.8) we notice that $H_{\varepsilon} \subset H_{\varepsilon^{\prime}} \subset X$, for $\varepsilon>\varepsilon^{\prime}>0$. So $\mu_{\varepsilon}$ is increasing in $\varepsilon$ and $\mu_{\varepsilon} \geqq \mu_{0}$. Therefore, as $\varepsilon \rightarrow 0, \lim \mu_{\varepsilon} \geqq \mu_{0}$. On the other hand, for each $w \in X$ with $\int w^{2} f d t=1$, let $w_{\varepsilon}=\eta_{\varepsilon} w$, where $\eta_{\varepsilon}$ is a continuous function such that $\eta_{\varepsilon}=0$ on $[0, \varepsilon]$ and $\left[\frac{\pi}{2}-\varepsilon, \frac{\pi}{2}\right], \eta_{\varepsilon}=1$ on $\left[2 \varepsilon, \frac{\pi}{2}-2 \varepsilon\right]$, and it is linear otherwise. Then $w_{\varepsilon} \in H_{\varepsilon}$, and one can check that $w_{\varepsilon} \rightarrow w$ in $X$ and $I\left(w_{\varepsilon}\right) \rightarrow I(w)$ as $\varepsilon \rightarrow 0$. But we have $I\left(w_{\varepsilon}\right) \geqq \mu_{\varepsilon} \int w^{2} f d t$. Letting $\varepsilon \rightarrow 0$ we get $I(w) \geqq \lim \mu_{\varepsilon}$. Since $w$ is taken arbitrarily, we see $\mu_{0} \geqq \lim \mu_{\varepsilon}$. This proves (2.8), and also the lemma.

Lemma 2.7. If $\mu_{0}>0$, then there exists $\delta>0$ such that

$$
I(v) \geqq \delta\|v\|^{2}, \quad \forall v \in X .
$$

Proof. Recall that in the expression of $I(v)$, the potential $Q(t)$ depends continuously on $\lambda=\left(\lambda_{1}, \lambda_{2}\right)$. Let $Q^{*}$ be the potential corresponding to $\lambda^{*}=(1-\delta)^{-1} \lambda$, and let $\mu_{0}^{*}$ be the corresponding eigenvalue, where $\delta>0$ is a sufficiently small constant. Then $\mu_{0}^{*}$ can be arbitrarily close to $\mu_{0}$. So we have

$$
\begin{aligned}
I(v) & =\delta \int \dot{v}^{2} f d t+(1-\delta) \int\left(\dot{v}^{2}+Q^{*} v^{2}\right) f d t \\
& \geqq \delta \int \dot{v}^{2} f d t+(1-\delta) \mu_{0}^{*} \int v^{2} f d t \geqq \delta\|v\|^{2} .
\end{aligned}
$$

The following is just the mountain pass theorem in our version.

Lemma 2.8. Suppose that $p>1$ and $\mu_{0}>0$. If $c_{0}<J(0)$, then there exists a solution $b$ of (2.1)-(2.3) with $J(b)>J(0)$.

Proof. By Lemma 2.2, $c_{0}<J(0)$ implies $c_{0}$ is achieved by some $a_{0}$ which is a solution to (2.1)-(2.3). We will use the trick employed in the proof of Lemma 2.1 to prove the existence of $b$.

Let $Y_{0}=\left\{a \in X: 0 \leqq a(t) \leqq a_{0}(t), t \in\left(0, \frac{\pi}{2}\right)\right\}$. For any $a \in X$ define $a^{*} \in Y_{0}$, by

$$
a^{*}(t)=\left\{\begin{array}{lll}
a_{0}(t), & \text { if } & a(t)>a_{0}(t), \\
a(t), & \text { if } & 0 \leqq a(t) \leqq a_{0}(t), \\
0, & \text { if } & a(t)<0 .
\end{array}\right.
$$

Then define $G: R \times R \rightarrow R$ by

$$
G(t, a)= \begin{cases}F\left(t, a_{0}(t)\right), & \text { if } \quad a>a_{0}(t), \\ F(t, a), & \text { if } 0 \leqq a \leqq a_{0}(t), \\ F(t, 0), & \text { if } a<0,\end{cases}
$$

where $F(t, a)$ is the same as in the proof of Lemma 2.1. Instead of $J$, we consider the functional

$$
J^{*}(a)=\int_{0}^{\frac{\pi}{2}}\left(\dot{a}^{2}+G(t, a)\right) f(t) d t .
$$


Since $p>1$ and $q>1$, both $J$ and $J^{*}$ are smooth on $X$. One easily checks $J\left(a^{*}\right)$ $=J^{*}\left(a^{*}\right)$ by the above definitions. On the other hand, we derive from the minimizing property of $a_{0}$ that

$$
J^{*}\left(a^{*}\right) \leqq J^{*}(a), \quad \forall a \in X .
$$

Let $\Gamma=\left\{\gamma \in C([0,1], X): \gamma(0)=0, \gamma(1)=a_{0}\right\}$ be the set of all continuous paths joining 0 and $a_{0}$ in $X$. Set

$$
\bar{c}=\inf _{\gamma \in \Gamma[0,1]} \max _{t[0,}^{*}(\gamma(t)) .
$$

We claim that $\bar{c}>J(0)$. Let $\gamma$ be any path in $\Gamma$. Note that $\gamma^{*}$ is also a path in $\Gamma$. By (2.9), $\max _{\gamma} J^{*} \geqq \max _{\gamma^{*}} J^{*}$. On the other hand, $\gamma^{*}(t) \in Y_{0}$ and we have $J^{*}\left(\gamma^{*}(t)\right)$ $=J\left(\gamma^{*}(t)\right)$. By Lemma 2.7 and the Taylor expansion,

$$
\begin{aligned}
J(a) & =J(0)+I(a)+o\left(\|a\|^{2}\right) \\
& \geqq J(0)+\delta\|a\|^{2}+o\left(\|a\|^{2}\right), \quad \text { as } \quad a \rightarrow 0 .
\end{aligned}
$$

Therefore, we may take $\varepsilon>0$ with $\varepsilon<\left\|a_{0}\right\|$ such that $J(a) \geqq J(0)+\delta_{1}$ for

$$
a \in S_{\varepsilon}=\{a \in X:\|a\|=\varepsilon\},
$$

where $\delta_{1}=\delta \varepsilon^{2} / 2$. It is clear that the path $\gamma^{*}$ must intersect with $S_{\varepsilon}$. It follows that $\max J^{*} \geqq J(0)+\delta_{1}$. Hence, $\max J^{*}>J(0)+\delta_{1}$ for all $\gamma \in \Gamma$, and by definition $\bar{c} \geqq J(0)+\delta_{1}$.

Now choose a minimizing sequence $\left\{\gamma_{i}\right\}$ in $\Gamma$ such that $\max J^{*} \rightarrow \bar{c}$ as $i \rightarrow \infty$. By passing to $\gamma_{i}^{*}$ if necessary, we may assume $\gamma_{i} \subset Y_{0}$. From the proof of the deformation lemma in critical point theory (cf. [P]), we know there exists $a_{i} \in \gamma_{i}$ and $b_{i} \in X$ such that $J^{*}\left(a_{i}\right)=\max _{\gamma_{i}} J^{*},\left\|a_{i}-b_{i}\right\| \rightarrow 0$ and $d J^{*}\left(b_{i}\right) \rightarrow 0$. Note $J^{*}\left(a_{i}\right) \rightarrow \bar{c}$, and hence $J^{*}\left(b_{i}\right) \rightarrow \bar{c}$. In the sequel we will prove that $\left\{b_{i}\right\}$ subconverges to some $b \in X$ (which means $J^{*}$ satisfies a sort of Palais-Smale condition). Then it is clear that $d J^{*}(b)=0$, i.e. $b$ is a critical point of $J^{*}$, and $J^{*}(b)=\bar{c}$. Since $a_{i} \in Y_{0}$ for each $i$, we see $b \in Y_{0}$. But $J^{*}$ and $J$ coincide on $Y_{0}$, so $b$ is also a critical point of $J$ with $J(b)$ $=\bar{c}>J(0)$. Clearly $b$ is not one of the two constant critical points 0 and $\frac{\pi}{2}$, since $J(0)$ $<J(b)$ and $\frac{\pi}{2} \notin Y_{0}$. It follows from [S] that $b$ is a solution to (2.1)-(2.3).

It remains to show the subconvergence of $\left\{b_{i}\right\}$. From $J\left(a_{i}\right) \leqq C$ and $a_{i} \in Y_{0}$ we derive that $\left\{a_{i}\right\}$ is bounded in $X$. Since $b_{i}-a_{i} \rightarrow 0$ in $X$, we see $\left\{b_{i}\right\}$ is bounded in $X$. Thus, some subsequence of $\left\{b_{i}\right\}$, still denoted by $\left\{b_{i}\right\}$, converges weakly to some $b$ in $X$. The weak convergence implies

$$
\int\left(b_{i}-b_{j}\right)^{2} f d t \rightarrow 0, \text { as } i, j \rightarrow \infty .
$$

[Unless otherwise specified, integrals will always be over $\left(0, \frac{\pi}{2}\right)$.] On the other hand,

$$
\begin{aligned}
o(1) & =\frac{1}{2} d J^{*}\left(b_{i}\right)\left(b_{i}-b_{j}\right)=\int\left[\dot{b}_{i}\left(\dot{b}_{i}-\dot{b}_{j}\right)+\frac{1}{2} \frac{\partial G}{\partial a}\left(t, b_{i}\right)\left(b_{i}-b_{j}\right)\right] f d t \\
& =\int \dot{b}_{i}\left(\dot{b}_{i}-\dot{b}_{j}\right) f d t+\int Q \sin a_{i} \cos a_{i}\left(a_{i}-a_{j}\right) f d t+o(1),
\end{aligned}
$$

where $o(1)$ denotes any quantity going to zero as $i, j \rightarrow \infty$. This is because $a_{i}-b_{i} \rightarrow 0$ in $X$ and (2.4) holds in our case, also because $a_{i}, a_{j} \in Y_{0}$ and $G\left(t, a_{i}\right)=F\left(t, a_{i}\right)$. 
Interchanging the roles of $i$ and $j$ in the above equality, we get another similar equality. Subtracting the second equality from the first one gives

$$
\int\left(\dot{b}_{i}-\dot{b}_{j}\right)^{2} f d t+\int Q\left(\sin a_{i} \cos a_{i}-\sin a_{j} \cos a_{j}\right)\left(a_{i}-a_{j}\right) f d t=o(1) .
$$

The absolute value of the second integral on the left is dominated by

$$
\begin{aligned}
\int|Q|\left(a_{i}-a_{j}\right)^{2} f d t & =\int_{0}^{\varepsilon}+\int_{\frac{\pi}{2}-\varepsilon}^{\frac{\pi}{2}}+\int_{\varepsilon}^{\frac{\pi}{2}-\varepsilon}|Q|\left(a_{i}-a_{j}\right)^{2} f d t \\
& \leqq \int_{0}^{\varepsilon}+\int_{\frac{\pi}{2}-\varepsilon}^{\frac{\pi}{2}}|Q|\left|a_{0}\right|^{2} f d t+C(\varepsilon) \int\left(a_{i}-a_{j}\right)^{2} f d t
\end{aligned}
$$

where $\varepsilon>0$ is small. The first two integrals on the right-hand side of the above inequality can be made arbitrarily small by taking $\varepsilon$ sufficiently small, then fixing $\varepsilon$ and letting $i, j$ be sufficiently large; the last integral can be arbitrarily small too. This shows $\int|Q|\left(a_{i}-a_{j}\right)^{2} f d t=o(1)$, hence $\int\left(\dot{b}_{i}-\dot{b}_{j}\right)^{2} f d t=o(1)$ too. This and $(2.10)$ then show $\left\{b_{i}\right\}$ converges strongly in $X$. The proof of Lemma 2.8 is completed.

The next lemma is a crucial observation.

Lemma 2.9. Suppose $a$ is any solution to (2.1)-(2.3), then $J(a)<J(0)$.

Proof. Note first that

$$
J(a)-J(0)=\int\left(\dot{a}^{2}+Q(t) \sin ^{2} a\right) f d t .
$$

Writing Eq. (2.1) in the following form:

$$
\frac{d}{d t}(f(t) \dot{a})=f(t) Q(t) \sin a \cos a
$$

we see that

$$
\int Q \sin ^{2} a f d t=\int \operatorname{tg} a d(f \dot{a})=\left.f \dot{a} \operatorname{tg} a\right|_{0} ^{\frac{\pi}{2}}-\int \dot{a}^{2} \sec ^{2} a f d t .
$$

By the asymptotic analysis for the solutions of (2.1)-(2.3) near $t=0$ and $\frac{\pi}{2}$ in $[\mathrm{S}]$, the first term on the right side of the above equality vanishes. It follows that

$$
J(a)-J(0)=\int\left(1-\sec ^{2} a\right) \dot{a}^{2} f d t<0 .
$$

The following lemma completes the proof of Theorem 1.1.

Lemma 2.10. Suppose that $\mu_{0} \geqq 0$. Then (2.1)-(2.3) has no solutions.

Proof. Consider first the case where $p>1$ and $\mu_{0}>0$. Suppose there is a solution $a$ of (2.1)-(2.3). By Lemma 2.9, $J(a)<J(0)$, which implies $c_{0}<J(0)$. Then Lemma 2.8 guarantees the existence of another solution $b$ of $(2.1)-(2.3)$ with $J(b)>J(0)$, contradicting Lemma 2.9. This proves the lemma in the case $p>1$ and $\mu_{0}>0$.

For the remaining case, the existence of a solution of (2.1)-(2.3) still implies $c_{0}<J(0)$ by Lemma 2.9. Note that the condition $c_{0}<J(0)$ is open, i.e. it is satisfied by the functional $J$ with nearby parameters $p, q, \lambda_{1}, \lambda_{2}$. However, we can find nearby parameters with $p>1$ and $\mu_{0}>0$, for which (2.1)-(2.3) has no solution and hence $c_{0} \geqq J(0)$, a contradiction. This proves the lemma completely. 


\section{Discussions}

We can apply Theorem 1.1 to get many new examples of symmetric harmonic maps between spheres. But we will not try to present a complete list of these. We would like only to point out the following interesting consequence of Theorem 1.1.

Corollary 3.1. Let $f: S^{p} \rightarrow S^{m}$ and $g: S^{q} \rightarrow S^{n}$ be two homogeneous harmonic polynomial maps with the same homogeneity $k$; then there exists a harmonic map homotopic to $f * g$.

Proof. We apply Theorem 1.1 to the case where $\lambda_{1}=k(k+p-1)$ and $\lambda_{2}=k(k+q-1)$. In such a case (1.2) is equivalent to $q \geqq p$. Thus we need only to verify (2.6) under the assumption $(q-1)^{2} \geqq 4 \lambda_{2}$, which implies $q>1$. By a simple computation we see (2.6) is equivalent to the following two inequalities:

$$
\begin{gathered}
(p-1)(q-1)+2\left(\lambda_{2}-\lambda_{1}\right)>0, \\
\left(\lambda_{1}+\lambda_{2}\right)^{2}>(p+q-2)\left[\lambda_{1}(q-1)-\lambda_{2}(p-1)\right] .
\end{gathered}
$$

For the present case, these can be reduced to (i) $(q-1)(p-1)+2 k(q-p)>0$, and (ii)' $(2 k+p+q-2)^{2}>(p+q-2)(q-p)$. Since $q \geqq p \geqq 1, q>1$, and $k>1$, (i) $)^{\prime}$ and (ii) are clearly true.

There is a class of homogeneous harmonic maps of homogeneity 2 arising from orthogonal multiplications (cf. [E-L], (8.4)). By Corollary 3.1, the join of any two maps in this class is homotopic to a harmonic map.

On the other hand, from the nonexistence part of Theorem 1.1 we also see the limitation of finding harmonic maps by Smith's constructions. Therefore, to accomplish the task of representing harmonically $\pi_{k}\left(S^{n}\right)$ ([Y], Problem Section, \#112), one has to seek for other approaches to the problem, either making use of the symmetries of the Euclidean spheres in a different way or exploring deeper results in analysis.

Note. After this paper was finished, Prof. J. Eells kindly informed the author that similar results had been obtained by V. Pettinati and A. Ratto in their recent paper "Existence and non-existence results for harmonic maps between spheres," using completely different methods.

\section{References}

[E-L] Eells, J., Lemaire, L.: A report on harmonic maps. Bull. Lond. Math. Soc. 10, 1-68 (1978)

[H] Hartman, P.: Ordinary differential equations. 2nd edn. Boston: Birkhäuser 1982

[P] Palais, R.: Lusternik-Schnirelmann theory on Banach manifolds. Topology 5, 115-132 (1966)

[R] Ratto, A.: Construction d'applications harmoniques de spheres eucliennes, C.R. Acad. Sci. Paris 304, Ser. I 185-196 (1987)

[S] Smith, R.T.: Harmonic mappings of spheres. Am. J. Math. 97, 364-385 (1975)

[Y] Yau, S.-T. (ed.): Seminar on differential geometry. Princeton, NJ: Princeton University Press 1982

Communicated by C. H. Taubes

Received January 29, 1988; in revised form May 12, 1988 
